\title{
ESTUDO DA ATIVIDADE FOTOCATALÍTICA DE FOTOCATALISADORES SUPORTADOS PREPARADOS A PARTIR DE MDF E PORONGO ${ }^{1}$
}

\author{
STUDY OF PHOTOCATALYTIC ACTIVITY OF SUPPORTED \\ PHOTOCATALYSTS PREPARED FROM MDF AND PORONGO WASTE
}

\begin{abstract}
Matheus Severo Schalenberger ${ }^{2}$, Mariéle Schaedler Nascimento ${ }^{3}$ e William Leonardo da Silva ${ }^{4}$
\section{RESUMO}

O presente trabalho tem como objetivo comparar a eficiência de diferentes suportes/catalisadores para aplicação em fotocatálise heterogênea para degradação de Rodamina B (RhB) com a utilização de bio-óleos provindos de biomassas de resíduos de fibra de média densidade (MDF) e porongo, oriundos da produção dos mesmos. Foram realizadas análises que corroboram com a atividade fotocatalítica das amostras, através de caracterizações como potencial zeta, espectroscopia de reflectância difusa (UV-vis) e análise elementar $(\mathrm{CHN})$. Entre os sistemas testados, o fotocatalisador hibrido do bio-óleo (MDF) com $\mathrm{SiO}_{2}+\mathrm{TiO}_{2}$ apresentou melhor atividade fotocatalítica como uma degradação do corante $\mathrm{RhB}$ de $660,39 \%\left(\mathrm{k}=0,0080 \mathrm{~min}^{-1}\right)$, seguida da amostra híbrida com o resíduo de porongo $\left(54,42 \%\right.$ e $\left.\mathrm{k}=0,0065 \mathrm{~min}^{-1}\right)$, após 120 minutos de reação sob radiação UV, enquanto nas mesmas condições o catalisador $\mathrm{P}_{25}-\mathrm{TiO}_{2}$ obteve uma degradação de $50,02 \%\left(\mathrm{k}=0,0010 \mathrm{~min}^{-1}\right)$. Por conseguinte, verificou-se que os resíduos de MDF e porongo apresentaram grande potencialidade para serem utilizados como precursores de fotocatalisadores para aplicação na degradação de poluentes orgânicos por fotocatálise heterogênea.

Palavras-chave: fotocatálise heterogênea; titânio; corantes; catalisadores.

\section{ABSTRACT)}

The present work aims to compare the efficiency of different supports / catalysts for application in heterogeneous photocatalysis for degradation of Rhodamine $B$, with the use of bio oils from biomass fibers of medium density $(M D F)$ and porongo, residues from the production of themselves. Analyzes were performed that corroborate the photocatalytic activity of the samples, through characterizations such as zeta potential, diffuse reflectance spectroscopy and elementary analysis (CHN).Among the systems tested, the hybrid photocatalyst of bio-oil $(\mathrm{MDF})$ with $\mathrm{SiO}_{2}+\mathrm{TiO}_{2}$ showed better photocatalytic activity as a degradation of the RhB dye of $6060.39 \%$ $\left(k=0.0080 \mathrm{~min}^{-1}\right)$, followed by the hybrid sample with the porongo residue $\left(54.42 \%\right.$ e $\left.k=0.0065 \mathrm{~min}^{-1}\right)$, after 120 minutes of reaction under UV radiation, while in the same conditions the catalyst $\mathrm{P} 25-\mathrm{TiO}_{2}$ obtained a degradation of $50.02 \%\left(k=0,0010 \mathrm{~min}^{-1}\right)$. Consequently, it was found that MDF and pore waste have great potential to be used with photocatalyst precursors, for application in the degradation of organic pollutants by heterogeneous photocatalysis.

Keywords: heterogeneous photocatalysis; titanium; dyes; catalysts.

\footnotetext{
${ }^{1}$ Trabalho de Iniciação Científica.

${ }^{2}$ Acadêmico do Curso de Engenharia Química - Universidade Franciscana - UFN. E-mail: matheus_severo7@hotmail.com

${ }^{3}$ Acadêmica do Curso de Engenharia Química - Universidade Franciscana - UFN. E-mail: mariele.sn@hotmail.com

${ }^{4}$ Orientador - Universidade Franciscana - UFN. E-mail: w.silva@ufn.edu.br
} 


\section{INTRODUÇÃO}

A utilização de biomassas justifica-se pela necessidade de se encontrar fontes alternativas para a produção de bioprodutos. Nesse sentido, o bio-óleo apresenta destaque como uma alternativa ecotecnológica (OLIVEIRA; BELARMINO; CORIOLANO, 2016), principalmente em relação àquele oriundo de resíduos sólidos industriais, despertando o interesse de pesquisas de sua estrutura e finalidade.

Atualmente, o resíduo de Fibra de Média Densidade (Medium Density Fiberboard, MDF) é considerado refugo de produção em diversas indústrias moveleiras, fonte em expansão como alternativa para fins ambientais e econômicos, as quais possuem problemas quanto a destinação final correta desse resíduo em virtude da inviabilidade técnica e econômica (SCHALENBERGER et al., 2018a). Além disso, tem-se o resíduo de porongo, uma curcubitácea originária da África que se encontra cultivada principalmente na região Sul do Brasil para a produção principalmente de cuias para chimarrão (LANGE et al., 2006). Durante seu processamento quantidades significativas de resíduos são geradas onde pouco é reutilizado, por exemplo, para produção de cuias cerca de $50 \%$ do material é incinerado ou triturado para produção de adubo (LAGO, 2013). Desta forma, as biomassas residuais de MDF e porongo apresentam-se como fontes para produção de bio-óleo.

Os diferentes tipos de biomassa exibem características química e estruturais distintas, gerando bio-óleos com classes de compostos qualitativamente e/ou quantitativamente diferentes, conforme seus processos de extração e termodegradação (MORAES, 2012). Visto isso, diversas técnicas podem ser empregadas para a caracterização desse material, como potencial zeta, determinação da energia de band-gap, análise elementar, poder calorífico superior e atividade fotocatalítica para aplicação em fotocatálise heterogênea para a degradação de poluentes orgânicos.

Nesse sentido, o presente trabalho tem como objetivo produzir bio-óleo oriundo de resíduos de MDF e porongo e investigar sua utilização como catalisador para avaliação da sua atividade fotocatalítica, sendo uma fonte promissora para tecnologias avançadas de tratamento, como os Processos Oxidativos Avançados (POAs), destacando a fotocatálise heterogênea.

\section{MATERIAL E MÉTODOS}

\section{PREPARAÇÃO DOS BIO-ÓLEOS}

Para a preparação dos bio-óleos, utilizou-se o procedimento de digestão em meio ácido das amostras de resíduos, maravalha de MDF e porongo, conforme adaptação da literatura (SCHALENBERGER; MALLMANN; SALAZAR, 2016). Assim, inicialmente, preparou-se uma solução de resíduo e ácido sulfúrico $\left(\mathrm{H}_{2} \mathrm{SO}_{4}\right.$, P.A., Sigma-Aldrich, 1:4 v/v), em um bloco digestor $\left(75^{\circ} \mathrm{C}\right)$. Após, o material obtido foi acondicionado em tubos falcon para posterior caracterização. Os tubos foram 
etiquetados para garantir a rastreabilidade tanto do bio-óleo obtido a partir da maravalha de MDF, quanto do bio-óleo obtido do resíduo de porongo.

\section{SÍNTESE DOS FOTOCATALISADORES}

Os fotocatalisadores dopados foram preparados a partir do bio-óleo de respectivos resíduos, MDF e porongo, impregnados com o precursor de metal fotoativo $\left(\mathrm{P} 25-\mathrm{TiO}_{2}\right)$, conforme adaptação da literatura (DA SILVA et al., 2016). Inicialmente, os bio-óleos sofreram um pré-tratamento a fim de deixá-los na forma sólida, por meio do suporte com $\mathrm{SiO}_{2}$ (Aldrich Chemistry, 0,007 $\mu \mathrm{m}$ ). Assim, $3 \mathrm{~g}$ de bio-óleo e 0,4 g de sílica foram agitados magneticamente (90 min), seguido de secagem $\left(100{ }^{\circ} \mathrm{C} / 960 \mathrm{~min}\right)$ (DeLeo, Brasil) e calcinação (450 ${ }^{\circ} \mathrm{C} / 240 \mathrm{~min}$ ). Para a impregnação dos bio-óleos suportados em sílica com $\mathrm{TiO}_{2}$ (Sigma-Aldrich, P.A.) foi preparada uma solução do bio-óleos suportado/ $\mathrm{TiO}_{2}(25 \% \mathrm{~m} / \mathrm{m})$, sob agitação contínua $\left(25^{\circ} \mathrm{C} / 90 \mathrm{~min}\right)$ e calcinação $\left(450{ }^{\circ} \mathrm{C} / 240 \mathrm{~min}\right.$, com taxa de aquecimento $\left.10^{\circ} \mathrm{C} \mathrm{min}{ }^{-1}\right)$.

\section{TÉCNICAS DE CARACTERIZAÇÃO}

Para a determinação da energia de banda proibida ou band gap (Eg) utilizou-se a espectroscopia de refletância difusa (DRS) em um espectrofotômetro UV-visível (Cary 100 Scan, UV-Vis Spectrophotometers, Estados Unidos) com acessório DRA-CA-301 (Labsphere, Estados Unidos) acoplado para a análise em modo de refletância difusa. Assim, uma pequena quantidade de amostra (cerca de $0,05 \mathrm{~g}$ ) foi dispersa sobre uma célula com janela de quartzo confeccionada para análise de DRS. Os espectros foram obtidos a temperatura ambiente, sendo utilizado o sal $\mathrm{BaSO}_{4}$ como referência e a faixa espectral estudada foi de 200 a $600 \mathrm{~nm}$ e as amostras não sofreram nenhum pré-tratamento. Desse modo, o espectro obtido (absorbância versus comprimento de onda) é transformado em função de Kubelka-Munk e, através da equação (1) encontra-se o valor da band gap.

$$
E=\text { h.c. } \lambda^{-1}
$$

Sendo:

h: Constante de Planck $\left(4,136 \times 10^{-15} \mathrm{eV} \mathrm{s}\right)$;

c: velocidade da luz $\left(2,998 \times 10^{17} \mathrm{~nm} \mathrm{~s}^{-1}\right)$;

$\lambda$ : comprimento de onda característico da amostra (em nm) obtido pela extrapolação da parte linear da curva absorbância versus comprimento de onda.

O potencial zeta (PZ) foi utilizado para determinação da carga superficial das amostras. Assim, as amostras foram inicialmente diluídas em água destilada e deionizada, para posterior mensuração, 
em triplicatas, em um equipamento Malvern Zetasizer ${ }^{\circledR}$ Nano (ZEN3600, Reino Unido) equipado com Dip cell (Malvern, Reino Unido), utilizando um laser He-Ne de $4 \mathrm{~mW}$ e modelo Smoluchowski.

A análise elementar $(\mathrm{CHN})$ do bio-óleo de ambos os resíduos utilizou o analisador Perkin Elmer M-CHNS/O modelo 2400 (Wellesley, MA, Estados Unidos), para, assim, determinar o Poder Calorífico Superior (PCS), conforme a Eq. (2) (TILLMANN, 1978).

$$
P C S=437,288 . C-305,869
$$

Onde:

PCS: pode calorífico superior $\left(\mathrm{MJ} \mathrm{kg}^{-1}\right)$;

C: composição de carbono (\%).

\section{SISTEMA FOTOCATALÍTICO}

Para acompanhamento da fotodegradação do corante industrial Rodamina B, utilizou-se um reator batelada, do tipo slurry, sob radiação UV, com duração de $120 \mathrm{~min}$, conforme adaptação da literatura (DA SILVA et al., 2016). Assim, acompanhou-se a fotodegradação por meio de amostragem ( $1 \mathrm{~mL})$, em determinados tempos $(0,5,15,30,45,60,75,90$ e $120 \mathrm{~min})$, onde foram centrifugadas (centrífuga refrigerada de banca digital, Cientec CT-5000R, Brasil) por 20 min com rotação de 5000 rpm, temperatura de $25^{\circ} \mathrm{C}$ e 1 min de aceleração/desaceleração. Ao final, as amostras foram diluídas (0,5 mL da amostra em $5 \mathrm{~mL}$ de água destilada). Para determinação da absorbância dessas amostras, cubetas de quartzo foram preenchidas, uma com água destilada e a outra com a amostra a ser analisada. A absorbância (Abs) foi relacionada à concentração por meio de uma curva de calibração, conforme a Eq. (3).

$$
A b s=0,0168 . C
$$

Para a cinética heterogênea da fotodegradação, pesquisas de Konstantinou e Albanis (2003), bem como de Gaya e Abdullah (2008), apontam que a degradação fotocatalítica de contaminantes orgânicos se ajusta ao modelo cinético de Langmuir-Hinshelwood (L-H) para reações unimoleculares. Conforme Eq. (4), a taxa de reação apresenta dependência com as concentrações do soluto, para sistemas onde o reagente encontra-se suficientemente diluído. Essa equação pode ser integrada, gerando a Eq. (5), onde as constantes ks e K são contidas em uma única constante k, denominada de velocidade específica da reação. 


$$
\begin{gathered}
-r_{i}=-\frac{d C_{i}}{d t}=k_{S} \cdot K \cdot C_{i} \\
\ln \frac{C_{i o}}{C_{i}}=k_{S} . K . t=k . t \text { ou } C_{i}=C_{i o} \cdot e^{-k t}
\end{gathered}
$$

Onde:

$-r_{i}=$ taxa da reação;

$k_{S}=$ velocidade específica da reação superficial

$C_{i}=$ concentração do reagente;

$K=$ constante de adsorção do reagente na superfície do fotocatalisador.

\section{RESULTADOS E DISCUSSÃO}

\section{CARACTERIZAÇÃO DOS FOTOCATALISADORES}

A Tabela 1 apresenta os resultados de caracterização (potencial zeta e energia de band gap) dos fotocatalisadores. Para fins de comparação utilizou-se o fotocatalisador comercial P25- $\mathrm{TiO}_{2}$.

Tabela 1 - Potencial zeta (PZ) dos bio-óleos e catalisadores dopados.

\begin{tabular}{lccc}
\hline \multicolumn{1}{c}{ Amostra } & Potencial Zeta $(\mathbf{m V})$ & $\mathbf{E g}(\mathbf{e V})$ & $\boldsymbol{\lambda}(\mathbf{n m})$ \\
\hline $\mathrm{P} 25-\mathrm{TiO}_{2}$ & $-24,0 \pm 0,20$ & 3,34 & 371,25 \\
Bio-óleo (MDF) $+\mathrm{SiO}_{2}$ & $-1,63 \pm 0,90$ & 7,23 & 171,51 \\
Bio-óleo (porongo) $+\mathrm{SiO}_{2}$ & $-3,84 \pm 0,55$ & 8,74 & 141,88 \\
Bio-óleo (MDF) $+\mathrm{TiO}_{2}+\mathrm{SiO}_{2}$ & $-4,81 \pm 0,32$ & 2,42 & 512,40 \\
Bio-óleo (porongo) $+\mathrm{TiO}_{2}+\mathrm{SiO}_{2}$ & $-7,18 \pm 0,27$ & 2,65 & 467,92 \\
\hline \multicolumn{2}{r}{ Fonte: Construção do autor. }
\end{tabular}

Conforme a Tabela 1, todas as amostras de bio-óleo apresentaram carga superficial negativa na faixa de $-1,63$ até $-7,18 \mathrm{mV}$, porém, comparando com o catalisador comercial $\left(\mathrm{TiO}_{2}\right)$, as amostras dopadas apresentaram menores (em módulo) valores de potencial zeta (PZ). Destaca-se que a amostra de bio-óleo oriundo do porongo com $\mathrm{TiO}_{2}$ e $\mathrm{SiO}_{2}$ apresentou o maior valor de $\mathrm{PZ}$, o que pode indicar que o mesmo apresenta maior atividade fotocatalítica, visto que a molécula de RhB caracteriza-se por apresentar uma carga positiva, ou seja, uma natureza catiônica (HIEMENZ; RAJAGOPALAN, 1997; HUNGER, 2003; DEBRASSI et al., 2011; SALLEH et al., 2011). Sendo assim, o corante RhB tende a adsorver sobre a superfície catalítica de todos os catalisadores preparados por meio das forças eletrostáticas atrativas, visto a compatibilidade de cargas superficiais negativas dos fotocatalisadores e a carga positiva da RhB, permitindo a degradação do poluente orgânico. 
Além disso, os valores de Eg dos bio-óleos variaram entre 2,42 a 8,74 eV. De acordo com Mcevoy e colaboradores (2013), a energia de band gap (Eg) está ligado a eficiência do processo fotocatalítico heterogêneo, sendo que, quanto menor o valor de Eg, menor a quantidade de energia necessária para a fotoativação do catalisador. Assim, a amostra bio-óleo oriunda da maravalha de MDF suportado com $\mathrm{TiO}_{2}$ apresentou o melhor resultado, visto que apresentou o menor valor de $\mathrm{Eg}(2,65 \mathrm{eV})$ entre os catalisadores.

A Tabela 2 apresenta os resultados da composição química e os respectivos valores de poder calorífico superior das amostras.

Tabela 2 - Parâmetros obtidos da análise elementar e poder calorífico.

\begin{tabular}{lcccc}
\hline \multicolumn{1}{c}{ Amostra } & $\mathbf{C ~ ( \% )}$ & $\mathbf{H ~ ( \% )}$ & $\mathbf{N ~ ( \% )}$ & $\mathbf{P C S}\left(\mathbf{M J} \mathbf{~ k g}^{-1}\right)$ \\
\hline Bio-óleo (MDF) $+\mathrm{SiO}_{2}$ & 34,56 & 4,66 & 2,56 & 14,81 \\
Bio-óleo (porongo) $+\mathrm{SiO}_{2}$ & 36,78 & 4,56 & 1,25 & 15,78 \\
Bio-óleo (MDF) $+\mathrm{TiO}_{2}+\mathrm{SiO}_{2}$ & 32,42 & 4,02 & 2,34 & 13,87 \\
\hline Bio-óleo (porongo) $+\mathrm{TiO}_{2}+\mathrm{SiO}_{2}$ & 12,34 & 3,89 & 1,20 & 5,10 \\
\hline
\end{tabular}

Fonte: Construção do autor.

Conforme a Tabela 2, verifica-se a variação entre o poder calorífico superior das amostras, entre 5,1 $\mathrm{MJ} \mathrm{kg}^{-1}$ e 15,78 $\mathrm{MJ} \mathrm{kg}^{-1}$ ), enquanto o catalisador comercial $\left(\mathrm{TiO}_{2}\right)$ não apresenta PCS, visto a ausência de carbono na sua composição, conforme a Eq. (2). Demirbas (2004) garante que o poder calorífico superior deve estar na faixa de $14 \mathrm{MJ} \mathrm{kg}^{-1}$ a $21 \mathrm{MJ} \mathrm{kg}^{-1}$, comparando ao poder calorífico superior de biomassas, num geral. Estudos feitos por Martins e colaboradores (2007) utilizaram a biomassa serragem de eucalipto (Eucalyptus grandis) para obtenção do bio-óleo, onde poder calorífico demonstrou valor médio de 4,013 $\mathrm{kcal} \mathrm{kg}^{-1}\left(0,017 \mathrm{MJ} \mathrm{k}^{-1}\right)$. Além disso, foram observados baixos valores para nitrogênio, que variam entre 1,2\% e 2,56\%. Segundo Martins e colaboradores (2007), valores baixos deste parâmetro favorecem a utilização do bio-óleo em processos e sistemas para obtenção de energia, visto que geram menor emissão de gases poluentes. Assim, conclui-se que os bio-óleos de MDF e porongo suportados em sílica apresentaram um bom rendimento frente a esses parâmetros.

\section{ATIVIDADE FOTOCATALÍTICA}

A Tabela 3 apresenta os resultados de atividade fotocatalítica frente a degradação do corante $\mathrm{RhB}$, sob radiação UV. 
Tabela 3-Velocidades específicas de reação (k) dos ensaios com RhB sob radiação UV.

\begin{tabular}{lcc}
\hline \multicolumn{1}{c}{ Amostra } & Degradação UV (\%) & $\mathbf{k}_{\mathbf{U V}}\left(\mathbf{m i n}^{-1}\right)$ \\
\hline $\mathrm{P} 25-\mathrm{TiO}_{2}$ & 50,02 & 0,0010 \\
\hline Bio-óleo (MDF) $+\mathrm{SiO}_{2}$ & 13,86 & 0,0011 \\
Bio-óleo (porongo) $+\mathrm{SiO}_{2}$ & 8,58 & 0,0008 \\
Bio-óleo (MDF) $+\mathrm{TiO}_{2}+\mathrm{SiO}_{2}$ & 60,39 & 0,0080 \\
Bio-óleo (porongo) $+\mathrm{TiO}_{2}+\mathrm{SiO}_{2}$ & 54,42 & 0,0065 \\
\hline
\end{tabular}

Fonte: Construção do autor.

Conforme a Tabela 3, foi possível verificar que o híbrido do bio-óleo (MDF) com $\mathrm{SiO}_{2}$ e $\mathrm{TiO}_{2}$ apresentou melhor desempenho na degradação da $\mathrm{RhB}\left(60,39 \%\right.$ e k = 0,0080 $\left.\mathrm{min}^{-1}\right)$, seguido do hibrido do bio-óleo (porongo) com $\mathrm{SiO}_{2}+\mathrm{TiO}_{2}\left(54,42 \%\right.$ e k $\left.=0,0065 \mathrm{~min}^{-1}\right)$. Além disso, o efeito da combinação dos óxidos (híbrido) promoveu alterações nas propriedades elementares, texturais e estruturais que afetaram positivamente na atividade fotocatalítica. Em termos de ampliação de escala, a velocidade específica apresenta uma influência direta frente à degradação de poluentes orgânicos, visto que ao aumentar a velocidade específica (k), aumenta-se a geração de radicais hidroxila, proporcionando um aumento na atividade fotocatalítica. Além disso, destaca-se que esse processo fotocatalítico depende de quantidade de moléculas de poluente adsorvidas soba superfície e de radicais hidroxila, até uma mineralização completa ou equilíbrio químico com o tempo (SCHALENBERGER et al., 2018b).

\section{CONCLUSÃO}

Com o presente trabalho foi possível verificar a aplicabilidade do bio-óleo de maravalha e porongo como precursores de fotocatalisadores, para possível aplicação fotocatálise heterogênea. A análise de carga superficial das amostras, indicou cargas negativas nas amostras preparadas, possibilitando uma maior compatibilidade com o corante cationica RhB. Para a energia de band gap (Eg), a amostra MDF com $\mathrm{SiO}_{2} \mathrm{e} \mathrm{TiO}_{2}$ apresentou o menor valor (2,42 eV), sendo mais facilmente fotoativado e melhorando a atividade fotocatalítica. A análise elementar concluiu que tanto o bio-óleo de porongo quando o de MDF suportados em sílica, obtêm melhores resultados quanto ao poder calorífico. Por fim, verificou-se que foi possível produzir fotocatalisadores suportados com bio-óleo de MDF e porongo para potencial aplicação na degradação de poluentes orgânicos, visto que combinações de óxidos (suportes comerciais) aumentaram significativamente a degradação do corante RhB sob radiação UV. 


\section{REFERÊNCIAS}

DA SILVA, W. L.; LANSARIN, M. A.; DOS SANTOS, J. H. Z.; SILVEIRA, F. Photocatalytic degradation of rhodamine $B$, paracetamol and diclofenac sodium by supported titania-based catalysts from petrochemical residue: effect of doping with magnesium. Water Science and Technology, v. 74, n. 10, p. $2370-2383,2016$.

DEBRASSI, A. et al. Removal of cationic dye from aqueous solution using N-benzyl-O-carboxymethylchitosan magnetic nanoparticles. Chemical Engineering Journal, v. 183, n. 1, p. 284-293, 2011. DEMIRBAS, A. Combustion characteristics of different biomass fuels. Progress in Energy and Combustion Science, v. 20, n. 2, p. 219-230, 2004.

GAYA, U.I.; ABDULLAH, A.H. Heterogeneous photocatalytic degradation of organic contaminants over titanium dioxide: A review of fundamentals, progress and problems. Journal of Photochemistry and Photobiology C: Photochemistry Reviews, v. 9, p. 1-12, 2008.

HIEMENZ, P. C.; RAJAGOPALAN, R. Principles of Colloid and Surface Chemistry. 3. ed. Estados Unidos: CRC Press, 1997.

HUNGER, K. Industrial dyes: chemistry, properties, applications. 3. ed. Weinheim: Wiley-VcH, 2003.

KONSTANTINOU, L.; ALBANIS, T. Photocatalytic transformation of pesticides in aqueous titanium dioxide suspensions using artificial and solar light: intermediates and degradation pathways. Applied Catalysis B: Environmental, v. 42, n. 4, p. 319-335, 2003.

LAGO, R. E. T. Dissertação de Mestrado, Universidade Federal do Rio Grande do Sul, Brasil, 2013. LANGE, L.C. et al. Tratamento de lixiviado de aterro sanitário por processo oxidativo avançado empregando reagente fenton. Engenharia Sanitária e Ambiental, v. 11, n. 2, p. 175-183, 2006.

MARTINS, A. F. et al. Caracterização dos produtos líquidos e do carvão da pirólise de serragem de eucalipto. Química Nova, v. 30, n. 4, p. 873-878, 2007.

MCEVOY, J. G.; CUI, W.; ZHANG, Z. Degradative and disinfective properties of carbon-doped anatase-rutile $\mathrm{TiO}_{2}$ mixtures under visible light irradiation. Catalysis Today, v. 207, n. 1, p. 191-199, 2013. 
MORAES, M. S. A. Avaliação da composição química de bio-óleos de pirólise de diferentes biomassas utilizando a cromatografia gasosa monodimensional e a bidimensional abrangente. 2012. 160 p. Tese (Doutorado em Química) - Programa de Pós-graduação em Química, Universidade Federal do Rio Grande do Sul, Porto Alegre, 2012.

SALLEH, M. A. M. et al. Cationic and anionic dye adsorption by agricultural and solid wastes: A comprehensive review. Desalination, v. 280, n. 1-3, p. 1-13, 2011.

SCHALENBERGER, M. S. et al. Avaliação das propriedades térmicas, catalíticas e antimicrobianas do resíduo de Fibra de Média Densidade (MDF). In: Salão de Iniciação Científica, 8, 2018, Santa Maria. Anais Salão de Iniciação Científica. Santa Maria: Universidade Franciscana, 2018a.

SCHALENBERGER, M. S. et al. Síntese, caracterização e atividade fotocatalítica de fotocatalisadores dopados na degradação do corante rodamina B. Disciplinarum Scientia. Série: Naturais e Tecnológicas, v. 19, n. 2, p. 249-266, 2018 b.

SCHALENBERGER, M. S.; MALLMANN, E. S.; SALAZAR, R. F. S. Avaliação da digestão ácida como pré-tratamento do resíduo de fibra de média densidade (MDF) para posterior acondicionamento. In: Salão de Iniciação Científica, 6, 2016, Santa Maria. Anais de Salão de Iniciação Científica. Santa Maria: Universidade Franciscana, 2016.

OLIVEIRA, M. H. R.; BELARMINO, T. N. N.; CORIOLANO, A. C. F. Bio-óleo: uma alternativa energética sustentável. Educação Ambiental em Ação, n. 58, ano XV. 2016. Disponível em: https:// bit.ly/3oeZwFI. Acesso em: 14 jan. 2019.

TILLMAN, D. A. Wood as an energy resource.1 ed. Nova Iorque: Academic Press, 1978. 
